\title{
Influência da Ordem de Execução de Exercícios Resistidos na Hipotensão Pós-exercício em Idosos Hipertensos
}

\author{
Influence of Resistance Exercises Order Performance on Post-exercise \\ Hypotension in Hypertensive Elderly
}

Paulo Roberto Jannig' Ana Claudia Cardoso' Eriberto Fleischmann Carla Werlang Coelho ${ }^{1,2}$

Tales de Carvalho ${ }^{2}$

1. Universidade da Região de Joinville (Univille).

2. Universidade do Estado de Santa Catarina (Udesc-CCT/Cefid)

\section{Endereço para correspondência:}

Carla Werlang Coelho

Campus Universitário Avelino

Marcante, s/n, Bom Retiro -

89223-100 - Joinville, SC.

E-mail: carla@joinville.udesc.br

Submetido em: 13/11/2008

Versão final recebida em: 14/04/2009

Aceito em: 14/05/2009

\section{RESUMO}

Existem evidências de que exercícios resistidos contribuem para o controle de hipertensão arterial sistêmica, porém, são necessários estudos que indiquem a melhor forma de utilizá-los. O objetivo deste estudo foi analisar a influência da ordem de execução de exercícios resistidos na hipotensão pós-exercício em idosos com hipertensão arterial bem controlada. A amostra foi composta por oito idosos com hipertensão arterial sistêmica bem controlada (quatro homens e quatro mulheres). No protocolo 1 (P1) foram realizados inicialmente três exercícios para membros superiores e, posteriormente, três exercícios para membros inferiores. No protocolo 2 (P2) a sequência foi inversa. Já no protocolo 3 (P3) os exercícios foram realizados de forma alternada. Todos os exercícios foram realizados em três séries de 12 RM. Após cada protocolo a pressão arterial (PA) foi verificada em intervalos de 10 minutos, até 60 minutos pós-exercício. Os dados obtidos foram analisados através da ANOVA de fator duplo e fator único com post-hoc de Tukey e teste $t$ de Student pareado com distribuição bicaudal $(p<0,05)$. Em relação ao repouso, as seis verificações de PA pós-exercício de P1 não apresentaram diferenças significativas; no P2 foram significantemente diferentes apenas as verificações de 20 e 40 minutos na PAS; no P3 foram observadas diferenças significativas em todas as seis verificações da PAS e nas de 10, 20, 30 e 60 minutos da PAD. A verificação de 20 minutos na PAD do P3 demonstrou-se significantemente diferente das de 20 minutos de P1 e P2. Conclui-se que a ordem de realização de exercícios resistidos em idosos com hipertensão arterial bem controlada influenciou na duração da resposta hipotensiva, mas não diretamente em sua magnitude.

Palavras-chave: pressão arterial, massa muscular, tratamento não farmacológico.

\section{ABSTRACT}

There is evidence that resistance training can help to control hypertension, although further studies are needed to show the best way to perform it. The objective of this study was to analyze the influence of the resistance exercises order performance in post-exercise hypotension in elderly with controlled hypertension. The study sample consisted of eight elderly with controlled hypertension (four men and four women). In exercise protocol 1 (P1), they performed three upper limb exercises and then three lower limb exercises. In exercise protocol 2 (P2) the sequence was reversed. In exercise protocol 3 (P3) the subjects performed the same exercises in an alternate way. All exercises were performed in three sets of 12RM. Blood pressure (BP) was measured after each protocol at every 10 min until 60 min post-exercise. The data obtained were assessed using two-way ANOVA with post-hoc Tukey and paired Student's $t$ test $(p<0.05)$. Concerning rest values, $P 1$ did not show statistically significance in any moment; however, in P2 at 20 and 40 min Systolic Blood Pressure (SBP) values were statistically significant. Moreover, P3 showed differences in all SBP moments and also in the 10,20, 30 and 60 min of Diastolic Blood Pressure (DBP). When all the moments in the three protocols were compared just in the 20 min moment, P3 showed statistically difference compared with P1 and P2. It was concluded that the performed order of resistance exercises in elderly with controlled hypertension has influenced on the duration, but not directly on its magnitude.

Keywords: Blood pressure, muscular mass, non-pharmacological treatment. 


\section{INTRODUÇÃO}

Inúmeros estudos sugerem a prática de exercícios físicos como forma de tratamento e controle da hipertensão arterial, demonstrando que uma única sessão de exercícios físicos pode gerar decréscimo temporário na pressão arterial (PA) após o término do exercício, fenômeno denominado hipotensão pós-exercício (HPE) ${ }^{(1-4)}$.

A HPE já foi amplamente relatada após sessões de exercícios aeróbicos $^{(3-8)}$. Mais recentemente, surgiram estudos procurando demonstrar esse efeito após exercícios resistidos ${ }^{(8-14)}$, sendo, entretanto, contraditórios $^{(4)}$ os resultados em normotensos ${ }^{(13,14)}$, parecendo, então, que sujeitos hipertensos são mais suscetíveis à ocorrência de $\operatorname{HPE}^{(5,12,15)}$. Os mecanismos responsáveis pela HPE, portanto, ainda não estão definitivamente estabelecidos ${ }^{(12,15-17)}$.

Segundo Bermudes et al. ${ }^{(13)}$, alguns dos fatores que podem influenciar na magnitude e na duração do efeito hipotensivo pós-exercício são os níveis pressóricos de repouso e o tipo, a duração e a intensidade do exercício. São fatores que possibilitam diferentes respostas no sistema cardiovascular, alterando de forma significativa a PA pós-exercício. Apesar das discordâncias entre estudos, há consenso de que sessões agudas de exercício podem ter o potencial de agir como um benefício não farmacológico no auxílio do tratamento da hipertensão(1,8,16).

Segundo Lizardo e Simões ${ }^{(10)}$, diferentes formas de exercícios resistidos resultam em HPE; contudo, as sessões envolvendo maior massa muscular, como os membros inferiores (MI), apresentam efeito hipotensor mais significativo e duradouro em relação aos exercícios que utilizam menor massa muscular, como os membros superiores (MS). Já MacDonald et al. ${ }^{(15)}$ relatam que a massa muscular não afeta diretamente a magnitude da hipotensão pós-exercício, mas pode influenciar na duração dessa resposta.

Em posicionamento oficial, o American College of Sports Medicine(1) ressalta a importância de incluir o treinamento resistido em um programa de prevenção, tratamento e controle da hipertensão arterial. Assim, confirma-se a relevância de pesquisar os efeitos hipotensivos após sessão de exercícios resistidos, realizados de distintas maneiras, em prol de prescrição mais adequada. Tendo em vista o exposto, o objetivo deste estudo foi analisar a influência da ordem de execução de exercícios resistidos na hipotensão pós-exercício em idosos com hipertensão arterial bem controlada.

\section{MÉTODOS}

A amostra foi composta por oito idosos com hipertensão arterial bem controlada (62,1 $\pm 3,1$ anos; 72,8 \pm 9,9kg; 161,0 \pm 8,0 cm) (média \pm desvio padrão), de ambos os gêneros (quatro homens e quatro muIheres), não obesos, sem experiência prévia em treinamento resistido. Todos faziam uso de pelo menos um fármaco anti-hipertensivo, sendo orientados a que não modificassem o uso da medicação em vigência durante o experimento.

Durante os dias de experimento, os sujeitos foram orientados a não ingerir cafeína, bebidas alcoólicas, não fumar pelo menos duas horas antes do experimento, não fazer uso excessivo de sal, não praticar atividades físicas extenuantes e tomar o medicamento anti-hipertensivo no horário habitual.

Os critérios de inclusão foram: a) não possuir alguma outra doença que pudesse comprometer a resposta cardiovascular ao exercício; e b) não apresentar dano muscular, articular ou ósseo que pudesse comprometer a execução parcial ou total de algum exercício proposto.

Todos os sujeitos foram voluntários ao participar deste estudo, tendo assinado um termo de consentimento livre e esclarecido de acordo com a resolução 196/96 do Conselho Nacional de Saúde, aprovado pelo Comitê de Ética em Pesquisa da Universidade da Região de Joinville. Este estudo contou com financiamento do Fundo de Apoio à Pesquisa da Universidade da Região de Joinville.
Todos os sujeitos fizeram três visitas ao laboratório, em dias não consecutivos, para realizar a mensuração da PA de repouso (método auscultatório utilizando esfigmomanômetro de mercúrio), sendo seguidas rigorosamente as orientações que constam nas $V$ Diretrizes Brasileiras de Hipertensão Arteria|(18). Nessas ocasiões, os sujeitos foram informados sobre todos os procedimentos que seriam utilizados durante o experimento. Após esse momento, foram encaminhados à sala de musculação onde realizaram um teste de 12 repetições máximas (RM) em ordem aleatória para cada um dos seguintes exercícios: 1) supino sentado na máquina; 2) puxada alta anterior; 3) remada rlta; 4) leg press 900; 5) extensão de joelhos; e 6) flexão de joelhos. Cada um dos sujeitos foi submetido a cinco tentativas para determinar a carga proporcional a 12 RM em cada exercício; entre cada tentativa foi dado um intervalo de descanso de três a cinco minutos.

Após realização dos testes de carga, os indivíduos foram submetidos aleatoriamente a três diferentes protocolos. Estes foram realizados num período de sete dias, partindo do dia de determinação das cargas relativas a 12 RM, com um intervalo de no mínimo 48 horas entre cada protocolo. No protocolo 1 (P1) os exercícios foram organizados na seguinte ordem: 1) leg press 90॰; 2) extensão de joelho; 3) flexão de joelho; 4) supino sentado na máquina; 5) puxada alta anterior; e 6) remada alta. Dessa forma, realizando primeiro todos os exercícios para Ml e, em seguida, todos para MS. No protocolo 2 (P2) a situação foi inversa, executando primeiros os três exercícios para membros superiores, seguidos dos três exercícios para membros inferiores. Já o protocolo 3 (P3) foi organizado de maneira a intercalar um exercício para MS com um para MI. Todos os exercícios foram realizados em um método de três séries de 12 RM, com um intervalo de dois a três minutos entre cada série e exercício. Após cada protocolo a pressão arterial foi verificada em intervalos de 10 minutos, até 60 minutos pós-exercício.

A PA de repouso durante o experimento foi definida como a média dos três dias de visita ao laboratório e a aferida no momento pré-exercício em cada protocolo.

Os dados obtidos foram analisados de forma estatística por análise de variância (ANOVA) de fator duplo e fator único com post-hoc de Tukey e teste $t$ de Student pareado com distribuição bicaudal. Os resultados foram considerados significantes ao apresentar $p<0,05$.

\section{RESULTADOS}

Os valores encontrados em repouso e nas verificações pós-exercício, tanto para a PAS quanto para PAD, nos distintos protocolos, estão nas figuras 1 e 2. A análise estatística demonstrou haver diferenças significativas entre os protocolos, tanto na PAS ( $p=0,000)$ como na PAD ( $p=0,002)$; o P3 diferiu significantemente de P1 e P2 em relação à PAS $(p=0,000)$ e à PAD $(p=0,004)$. Contudo, P1 e P2 não diferiram entre si em relação à PAS $(p=0,599)$ e à PAD $(p=1,000)$.

Observa-se na figura 1 que P1 não apresentou diferenças significativas em relação ao repouso quanto à PAS em nenhuma das seis verificações de pressão arterial pós-exercício. Dessa maneira, não ocorreu hipotensão pós-exercício no P1. Já no P2, ocorreram diferenças nas verificações de 20 e 40 minutos de recuperação (20 min: $p=0,001$; 40 min: $p=0,033$ ), ocorrendo, assim, leve hipotensão pós-exercício. No P3, foram encontradas diferenças em todas as verificações após a realização da sessão de exercícios resistidos (10 min: $p=0,005 ; 20$ min $p=0,008 ; 30$ min: $p=0,008 ; 40$ min: $p=0,009 ; 50$ min: $p=0,017 ; 60$ min: $p=0,027)$. Assim, o P3 demonstrou-se extremamente eficaz em produzir hipotensão pós-exercício.

De acordo com a figura 2, tanto P1 quanto P2 não apresentaram diferenças significativas em relação ao repouso quanto à PAD em nenhum momento da recuperação. Dessa maneira, não ocorreu hipotensão diastólica pós-exercício no P1 e P2. No entanto, nas verificações de 


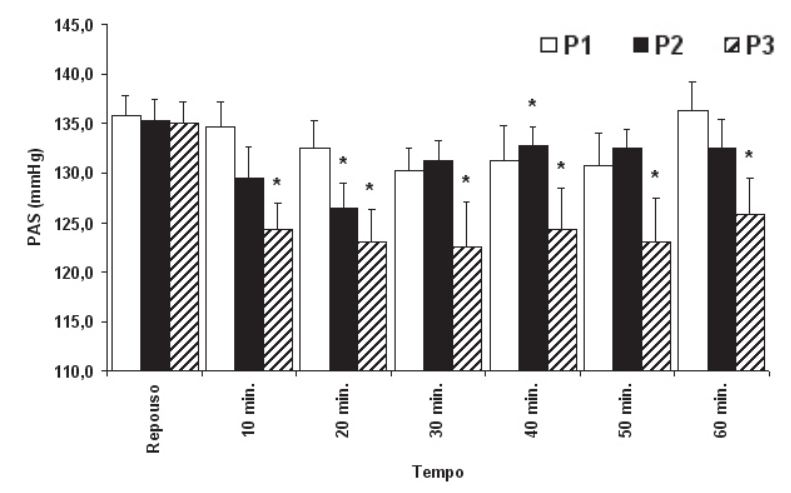

Figura 1. Estratificação dos valores da pressão arterial sistólica (PAS) nos respectivos tempos e protocolos. Valores em média (erro padrão da média); P1 = protocolo 1; $\mathrm{P} 2$ = protocolo $2 ; \mathrm{P} 3=$ protocolo $3 ; \mathrm{min} .=$ minuto; ${ }^{*}$ indica $\mathrm{p}<0,05$ em relação ao nível de repouso.

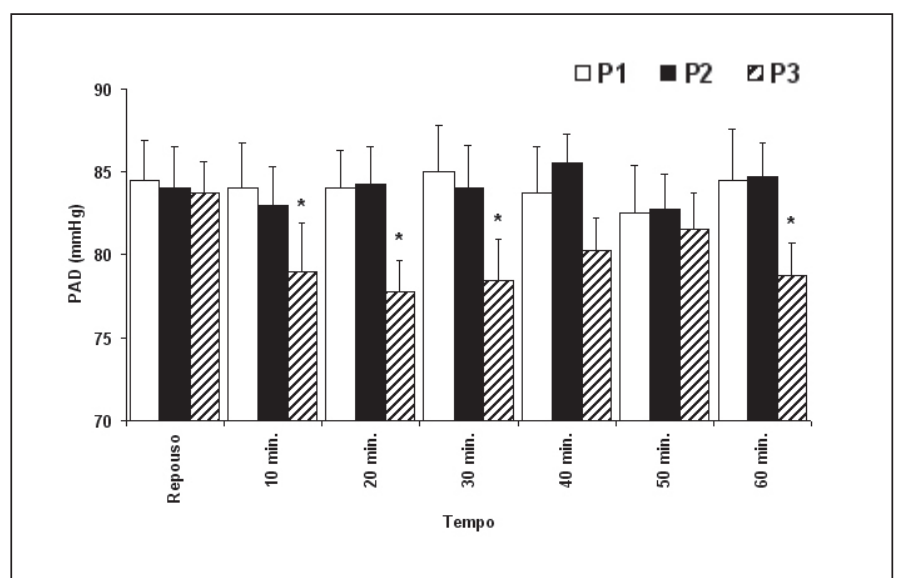

Figura 2. Estratificação dos valores da pressão arterial diastólica (PAD) nos respectivos tempos e protocolos. Valores em média (erro padrão da média); P1 = protocolo 1; $\mathrm{P} 2$ = protocolo $2 ; \mathrm{P} 3=$ protocolo $3 ;$ min. $=$ minuto; ${ }^{*}$ indica $\mathrm{p}<0,05$ em relação ao nível de repouso.

20, 30 e 60 minutos pós-exercício do P3, foram observadas diferenças significativas em relação aos níveis de repouso (20 min: $p=0,000 ; 30$ min: $p=0,001 ; 60$ min: $p=0,028)$, correspondendo também a leve hipotensão diastólica pós-exercício.

Para confirmação dos dados encontrados nas figuras 1 e 2, fez-se necessário, como no estudo de MacDonald et al. ${ }^{(15)}$, realizar uma análise da pressão arterial relativa aos valores em repouso, utilizando-se valores em delta (pressão arterial pós-exercício menos pressão arterial de repouso). Ao realizar tal análise (figuras 3 e 4), foram encontrados os mesmos valores anteriores, tanto para PAS como para PAD, confirmando os resultados já citados. No P1, P2 e P3 houve decréscimo médio da PAS durante o período de recuperação de 3,2 \pm 8,8, 4,5 $\pm 6,7$ e 11,3 \pm $9,1 \mathrm{mmHg}$, respectivamente. Já a PAD apresentou os seguintes decréscimos: $0,5 \pm 5,6,0,0 \pm 4,4$ e 4,4 $\pm 4,5 \mathrm{mmHg}$, respectivamente.

Ainda, através dessa análise em delta, foi constatada a diferença do decréscimo da pressão arterial na comparação entre os protocolos, demonstrando as eventuais diferenças quanto à magnitude da hipotensão pós-exercício. Os resultados em delta da PAS não demonstraram haver diferenças significativas entre os respectivos protocolos. Contudo, verificando os resultados em delta da PAD, o momento de 20 minutos pós-exercício no P3 mostrou-se significantemente diferente do mesmo momento no P1 e P2 $(p=0,008)$.

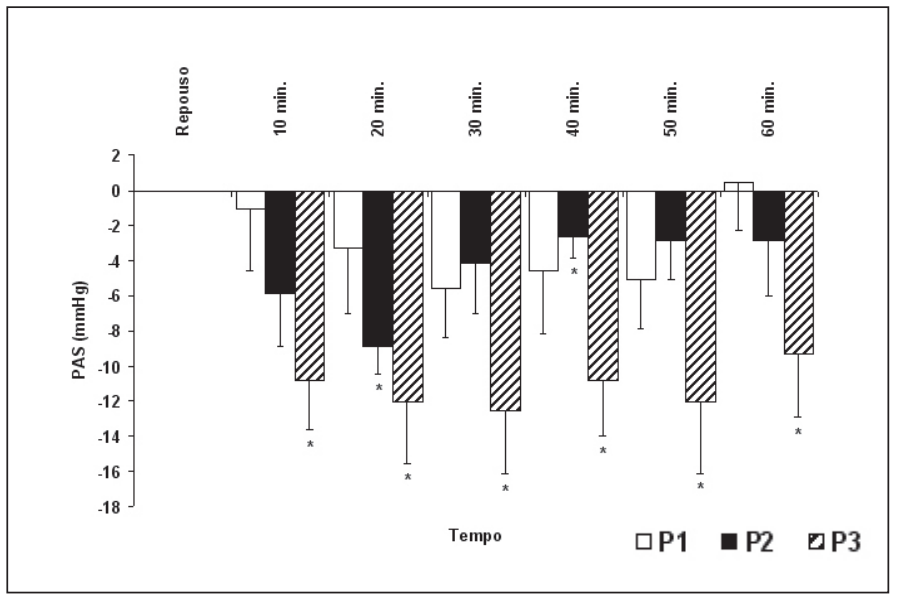

Figura 3. Diferença entre os valores da pressão arterial sistólica (PAS) pós-exercício e de repouso nos três diferentes protocolos de exercícios resistidos. Valores em média (erro padrão da média); $\mathrm{P} 1=$ protocolo $1 ; \mathrm{P} 2=$ protocolo $2 ; \mathrm{P} 3=$ protocolo $3 ; \mathrm{min} .=$ minuto; * indica $p<0,05$ em relação ao nível de repouso.

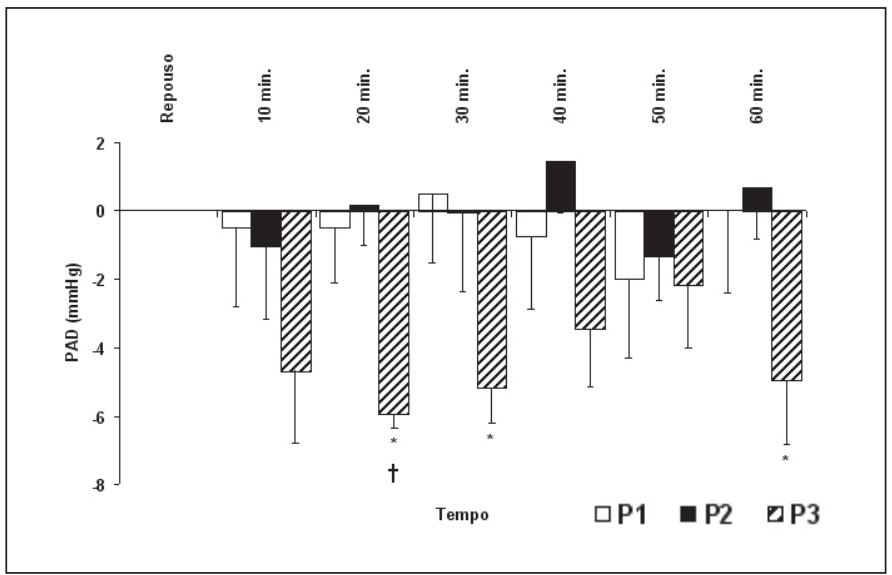

Figura 4. Diferença entre os valores da pressão arterial diastólica (PAD) pós-exercício e de repouso nos três diferentes protocolos de exercícios resistidos. valores em média (erro padrão da média); $\mathrm{P} 1=$ protocolo $1 ; \mathrm{P} 2=$ protocolo $2 ; \mathrm{P} 3=$ protocolo $3 ; \mathrm{min}$. = minuto; * indica $p<0,05$ em relação ao nível de repouso; $\uparrow$ indica $p<0,05$ em relação ao $P 1$ e $P 2$ no mesmo tempo.

\section{DISCUSSÃO}

Os principais resultados encontrados foram: 1) a ordem de execução de exercícios resistidos para Ml e MS influencia na HPE em idosos com hipertensão arterial sistêmica bem controlada; 2) existe maior duração da resposta hipotensiva como decorrência de uma sessão de exercícios resistidos alternados por segmentos (MI versus MS); 3) contudo, essa alternância por segmentos não parece influenciar na magnitude da $\mathrm{HPE}$; 4) a PAS demonstrou-se mais suscetível à HPE do que a PAD, com exceção de que apenas a PAD, em um momento, apresentou diferenças na magnitude de redução da PA; e 5) a HPE decorre da redução do fluxo simpático ao miocárdio.

Conforme Lizardo e Simões ${ }^{(10)}$, exercícios resistidos para MI proporcionam a ativação de quantidade de massa muscular maior do que exercícios resistidos para MS, mesmo sendo realizados na mesma intensidade. Esse fato se deve à distribuição da massa muscular no corpo humano, pois se sabe que os MS possuem massa muscular relativamente inferior à dos MI. Dessa maneira, a resistência vascular sistêmica sofre maior queda após exercícios para Ml, já que, durante a realização de algum exercício, ocorre vasodilatação dos vasos sanguíneos da musculatura ativa, com vasoconstrição da musculatura inativa ${ }^{(15-17)}$. 
Considerando os fatos citados acima, exercícios resistidos que envolvam a utilização de massa muscular menor (por exemplo, MS) provocarão também menor aumento da PA durante a execução, fato devido à pequena rede vascular que estará ocluída ${ }^{(16)}$. Assim, após o término do exercício, uma quantidade pequena de vasos se encontrará dilatada. Inversamente, exercícios resistidos para Ml induzirão picos de PA mais elevados durante o exercício; contudo, a quantidade de vasos em dilatação nos momentos pós-exercício será maior. Como ocorrido no P1 do presente estudo, ao final da sessão com três exercícios para MS, a resposta da pressão arterial pós-exercício não demonstrou haver queda significativa da mesma. Isso sugere, então, que a musculatura inativa (MI) durante a realização dos exercícios para MS continuou em estado de vasoconstrição nos momentos de recuperação e a vasodilatação da musculatura ativa (MS) não foi suficiente para gerar um efeito hipotensivo significativo nos momentos pós-exercício.

Ao observar o P2, que demonstrou a ocorrência de HPE em alguns momentos, sugere-se então que essa queda da pressão arterial se deve ao fato de que a musculatura inativa (MS) era relativamente menor em consideração à musculatura ativa (MI), já que os três últimos exercícios realizados foram para MI.

Contudo, P3 demonstrou-se extremamente eficaz em produzir a HPE através de exercícios realizados com alternância de membros. Uma das hipóteses para esse caso é de que, ao realizar os exercícios com alternância de membros, ocorra maior mobilização vascular, fazendo com que haja vasodilatação sistêmica, com pouca resposta vasoconstritora.

Em linhas gerais, a PAS respondeu de forma mais acentuada à HPE do que a PAD. Esse fato deve-se talvez a redução maior do fluxo nervoso simpático ao músculo cardíaco (diminuindo a contratilidade do miocárdio, ocorrendo então diminuição do volume de ejeção do ventrículo esquerdo e, consequentemente, do débito cardíaco), do que um decréscimo da resistência periférica total ${ }^{(5,17-21)}$. Neste estudo, isso pode ter ocorrido devido ao processo de envelhecimento, que faz com que as artérias respondam de forma insatisfatória às substâncias vasodilatadoras (arteriosclerose) e/ou essas substâncias passam a ser produzidas em menores quantidades ${ }^{(22,23)}$.

Ao estudar a resposta hipotensiva após exercício aeróbico em idosos hipertensos, Hagberg et al. ${ }^{(20)}$ encontraram decréscimos significativos na PAS, mas a PAD não apresentou diferenças, sugerindo que esse decréscimo ocorreu devido à redução do débito cardíaco,

\section{REFERÊNCIAS BIBLIOGRÁFICAS}

1. Pescatello LS, Franklin BA, Fagard R, Farquhar WB, Kelley GA, Ray CA. American College of Sports Medicine position stand. Exercise and hypertension. Med Sci Sports Exerc. 2004;36:533-53.

2. Kenney MJ, Seals DR. Postexercise hypotension. Key features, mechanisms, and clinical significance. Hypertension. 1993;22:653-64.

3. MacDonald JR, MacDougall JD, Hogben, CD. The effects of exercise duration on post-exercise hypotension. J Hum Hyperten. 2000;14:125-9.

4. MacDonald JR, MacDougall JD, Hogben, CD. The effects of exercise intensity on post-exercise hypotension. J Hum Hyperten. 1999;13:527-31.

5. Rondon MUPB, Alves MJ, Braga AM, Teixeira OT, Barretto AC, Krieger EM, et al. Postexercise blood pressure reduction in elderly hypertensive patients. J Am Coll Cardiol. 2002;39:676-82.

6. Forjaz CLM, Santaella DF, Rezende LO, Barretto ACP, Negrão CE. A duração do exercício determina a magnitude e a duração da hipotensão pós-exercício. Arq Bras Cardiol. 1998;70:99-104.

7. Senitko AN, Charkoudian N, Halliwill JR. Influence of endurance exercise training status and gender on postexercise hypotension. J Appl Physiol. 2002;92:2368-74

8. MacDonald JR, MacDougall JD, Interisano SA, Smith KM, McCartney N, Moroz JS, et al. Hypotension following mild bouts of resistance exercise and submaximal dynamic exercise. Eur J Appl Physiol. 1999;79:148-54.

9. Simão R, Fleck SJ, Polito M, Monteiro W, Farinatti P. Effects of resistance training intensity, volume, and session format on the postexercise hypotensive response. J Strength Cond Res. 2005;19:853-8.

10. Lizardo JHF, Simões HG. Efeitos de diferentes sessões de exercícios resistidos sobre a hipotensão pósexercício. Rev Bras Fisioter. 2005;9:289-95.

11. Polito MD, Simão R, Senna GW, Farinatti PTV. Efeito hipotensivo do exercício de força realizado em intensidades diferentes e mesmo volume de trabalho. Rev Bras Med Esporte. 2003;9:69-73. já que a resistência periférica total estava aumentada nos períodos pós-exercício. Fisher ${ }^{(21)}$, em estudo realizado tanto em mulheres de meia-idade normotensas quanto em hipertensas, demonstrou que uma única sessão de exercícios resistidos foi capaz de provocar leve resposta hipotensiva sistólica durante o período de recuperação. Assim, parece que a hipotensão sistólica aguda encontrada no presente estudo foi em decorrência de exercícios resistidos em ordem de alternância de membros, podendo ter ocorrido através de redução do fluxo simpático ao coração e não queda da resistência periférica total; contudo, necessita-se investigar esses mecanismos. Vale ressaltar que ao analisar esse mesmo efeito realizado de forma crônica, a tendência seja de que ocorrerá redução da resistência periférica total(1).

Ao se avaliar a magnitude da HPE nos três diferentes protocolos utilizados, encontrou-se que a PAS não demonstrou diferenças significativas na magnitude da resposta. Contudo, a PAD apresentou uma diferença considerável no $20^{\circ}$ minuto do $\mathrm{P} 3$ em relação ao mesmo momento do P1 e P2. Uma das hipóteses para esse ocorrido é de que o P3 possibilitou maior redução da resistência periférica total.

Assim, os resultados do presente estudo corroboram os encontrados por MacDonald et al.(15), que relatam que a massa muscular trabaIhada não afeta diretamente a magnitude da hipotensão pós-exercício, mas pode influenciar na duração dessa resposta. Lizardo e Simões ${ }^{(10)}$ também sugerem que diferentes formas de exercícios resistidos resultam em HPE; contudo, as sessões envolvendo maior massa muscular, como os membros inferiores, apresentam um efeito hipotensor mais significativo e duradouro em relação a exercícios que utilizem menor massa muscular, como os membros superiores.

Ressalta-se a importância clínica dos resultados encontrados, já que no P3 ocorreu redução média da PAS e PAD de 11,3 \pm 9,1 e 4,4 $\pm 4,5 \mathrm{mmHg}$, respectivamente. Decréscimos de aproximadamente $2 \mathrm{mmHg}$ na PAS e na PAD reduzem os riscos de acidente vascular cerebral em 14\% e 17\%, e risco de doença arterial coronariana em $9 \%$ e $6 \%$, respectivamente ${ }^{(1)}$.

Tendo como principal objetivo analisar a influência da ordem de execução de exercícios resistidos na hipotensão pós-exercício em idosos hipertensos controlados, pode-se concluir que: 1) exercícios resistidos proporcionam HPE em idosos com pressão arterial bem controlada; 2) a ordem de execução de exercícios resistidos influencia na duração da HPE, mas não na magnitude dessa resposta.
12. Mediano MFF, Paravidino V, Simão R, Pontes FL, Polito MD. Comportamento subagudo da pressão arteria após o treinamento de força em hipertensos controlados. Rev Bras Med Esporte. 2005;11:337-40.

13. Bermudes AMLM, Vassallo DV, Elisardo Corral Vasquez EC, Lima EG. Monitorização ambulatorial da pressão arterial em individuos normotensos submetidos a duas sessões únicas de exercício: resistido e aeróbio. Arq Bras Cardiol. 2003:82:57-64

14. Roltsch MH, Mendez T, Wilund KR, Hagberg JM. Acute resistive exercise does not affect ambulatory blood pressure in young men and women. Med Sci Sports Exerc. 2001;33:881-6.

15. MacDonald JR, MacDougall JD, Hogben CD. The effects of exercising muscle mass on post exercise hypotension. J Hum Hypertens. 2000;14:317-20.

16. MacDougall JD, Tuxen D, Sale DG, Moroz JR, Sutton JR. Arterial blood pressure response to heavy resistance exercise. J Appl Physiol. 1985;58:785-90

17. Halliwill JR. Mechanisms and clinical implications of post-exercise hypotension in humans. Exerc Sport Sci Rev. 2001;29:65-70

18. V Diretrizes Brasileiras de Hipertensão Arterial. Rev Hipertensão. 2006;9:121-56.

19. MacDonald JR. Potential causes, mechanisms, and implications of post exercise hypotension. J Hum Hypertens. 2002;16:225-36.

20. Hagberg JM, Montain SJ, Martin WH 3rd. Blood pressure and hemodynamic responses after exercise in older hypertensives. J Appl Physiol. 1987;63:270-6.

21. Fisher MM. The effect of resistance exercise on recovery blood pressure in normotensive and borderline hypertensive women. J Strength Cond Res. 2001;15:210-6.

22. Guyton AC, Hall JE. Tratado de fisiologia médica. 11 ed. Rio de Janeiro: Elsevier, 2006.

23. Taaffe DR, Galvão DA, Sharman JE, Coombes JS. Reduced central blood pressure in older adults following progressive resistance training. J Hum Hypertens. 2007;21:96-8. 